\title{
Examining the Association Between Serum Leptin and Sarcopenic Obesity
}

\author{
Zhe-Yu Yang ${ }^{1,2}$ \\ Wei-Liang Chen (D) ${ }^{2-4}$ \\ 'Department of General Medicine, Tri- \\ Service General Hospital; and School of \\ Medicine, National Defense Medical \\ Center, Taipei, Taiwan, Republic of China; \\ ${ }^{2}$ Division of Family Medicine, \\ Department of Family and Community \\ Medicine, Tri-Service General Hospital; \\ and School of Medicine, National \\ Defense Medical Center, Taipei, Taiwan, \\ Republic of China; ${ }^{3}$ Division of Geriatric \\ Medicine, Department of Family and \\ Community Medicine, Tri-Service \\ General Hospital; and School of \\ Medicine, National Defense Medical \\ Center, Taipei, Taiwan, Republic of China; \\ ${ }^{4}$ Department of Biochemistry, National \\ Defense Medical Center, Taiwan, \\ Republic of China
}

Objective: Sarcopenic obesity is an emerging geriatric syndrome among elderly individuals. Studies revealed a complicated pathogenesis between sarcopenia and obesity. Leptin, a proinflammatory adipokine, has been implicated in the mechanism of sarcopenic obesity. This study investigated the relationship between serum leptin level and sarcopenic obesity. Methods: The study included 4063 participants aged 60 years and older from the NHANES III database. Sarcopenia was defined as a skeletal muscle index (SMI) less than one standard deviation below the young adult value. Obesity was defined as a body mass index (BMIF) over $30 \mathrm{~kg} / \mathrm{m}^{2}$. Multivariate regression analysis was performed to examine the association between serum leptin level and sarcopenic obesity.

Results: In adjusted models, serum leptin level was positively correlated with BMI ( $\beta$ : 1.33, $\mathrm{p}$ value $<0.001)$ and negatively correlated with SMI $(\beta:-0.091, \mathrm{p}$ value $=0.001)$. A significant association between serum leptin level and sarcopenic obesity was found in multivariate analysis $(\beta: 4.011, \mathrm{p}$ value $=0.014)$.

Conclusion: Our study demonstrated that serum leptin level was related to an increased risk of sarcopenic obesity. This epidemiologic finding suggests that leptin may play a role in sarcopenic obesity.

Keywords: serum leptin levels, sarcopenia, sarcopenic obesity

\section{Introduction}

Adipose tissue, an endocrine organ, secrets various adipokines that modulate energy hemostasis and neuroendocrine function. ${ }^{1}$ Leptin, a kind of adipokine, is secreted by white adipose tissue and is correlated with total fat mass. ${ }^{2}$ Leptin acts on the hypothalamus to regulate appetite and food consumption. ${ }^{3}$ Moreover, leptin exerts effects on peripheral organs such as pancreatic beta cells, immune cells and muscle cells which are involved in glucose metabolism, inflammatory processes and insulin resistance. ${ }^{4,5}$ Tilg et al demonstrated that leptin stimulates the production of proinflammatory cytokines, including tumor necrosis factor- $\alpha$ (TNF- $\alpha$ ), interleukin-6 (IL-6), and interleukin-12 (IL-12). ${ }^{6}$ The presence of an inflammatory response has been proposed to be associated with diabetes mellitus, obesity, atherosclerosis and sarcopenia. ${ }^{6-8}$

Obesity, well recognized as a systemic inflammatory disorder, is correlated with numerous chronic diseases, such as hypertension, diabetes mellitus and cardiovascular diseases. ${ }^{9}$ An increased prevalence of obesity among elderly individuals in the US was shown by Flegal et al. ${ }^{10}$ Obesity-associated conditions have been found to contribute to the occurrence of sarcopenia. ${ }^{11}$ Sarcopenia, a geriatric syndrome, is an age-associated decline in muscle mass, quality and physical performance. ${ }^{12}$ The rising prevalence of sarcopenia has been reported worldwide. ${ }^{13}$ The combination of obesity and sarcopenia,
Division of Geriatric Medicine, Department of Family Medicine, Tri-Service General Hospital, National Defense Medical Center, Number 325, Section 2, Chang-gong Road, Nei-Hu District, I 14, Taipei, Taiwan

Tel +886-2-879233II ext. 16567

Fax +886-2-87927057

Email weiliang0508@gmail.com 
called sarcopenic obesity, was initially proposed by $\mathrm{RN}$. Baumgartner. ${ }^{14}$ Sarcopenic obesity is associated with elevated risks of disability, frailty, hospitalization and mortality in the older population. ${ }^{12}$ Sarcopenia and obesity have complex interactions and share a common etiology, including oxidative stress, proinflammatory cytokines, insulin resistance, hormonal alteration and functional impairment. ${ }^{15}$ The inflammatory state contributed to by cytokines and adipokines plays a crucial role in the development of sarcopenic obesity. ${ }^{16}$

Due to the proinflammatory properties of leptin, ${ }^{6}$ previous studies have investigated the relationship between serum leptin level and sarcopenic obesity, but they have come to contradictory conclusions. In this study, we performed a cross-sectional analysis of 4062 participants to examine the association between serum leptin level and sarcopenic obesity.

\section{Methods}

\section{Study Population}

The National Center for Health Statistics (NCHS) of the Centers for Disease Control and Prevention conducted the third National Health and Nutrition Examination Survey (NHANES III) between October 1988 and October 1994. NHANES III was designed as a stratified multistage sample of noninstitutionalized US civilian representative of the US population. It contained sociodemographic information, medical examination and records and laboratory data. The Institutional Review Board of the NCHS approved the NHANES study protocols. Written informed consent was obtained from all subjects. All data analyzed in this study are de-identified information and publicly available on the NHANES website (https://wwwn.cdc. gov/nchs/nhanes/nhanes3/datafiles.aspx). We performed a cross-sectional analysis of the NHANES III database. We excluded subjects who were less than 60 years old, subjects without laboratory data (leptin, C-reactive protein (CRP) and insulin), and subjects without information on body mass index (BMI), waist circumference, thigh skinfold, skeletal muscle index (SMI) and gait speed. A total of 4063 eligible participants were enrolled in this study.

\section{Leptin Measurement}

Serum leptin specimens were collected in the morning after an 8-hour fast and stored at $-70^{\circ} \mathrm{C}$. Serum leptin measurement was conducted by Linco Research, Inc. by radioimmunoassay. The detectable levels of leptin were 0.5 to $100 \mathrm{mg} / \mathrm{L}$. (coefficients of variations: 3.4 to $8.3 \%$ )

\section{Body Composition}

Body weight was measured in kilograms using a digital scale, and body height was measured in centimeters $(\mathrm{cm})$ using a stadiometer. Waist circumference was measured to the nearest $0.1 \mathrm{~cm}$ around the horizontal level at the high point of the iliac crest. The thigh skinfold was measured to the nearest 0.1 millimeters ( $\mathrm{mm}$ ) using Holtain calipers (Holtain Ltd, Crymych, United Kingdom) at the midpoint of the thigh. Body measurements were conducted according to a standardized protocol. BMI was calculated as the body weight in kilograms divided by the square of the body height in meters $\left(\mathrm{kg} / \mathrm{m}^{2}\right)$. We defined obesity as BMI over $30 \mathrm{~kg} / \mathrm{m}^{2}$.

In NHANES III, bioelectrical impedance was measured by using a Valhalla 1990B Bio-Resistance Body Composition Analyzer (Valhalla Scientific, San Diego, CA, USA). We used bioelectrical impedance to estimate skeletal muscle mass based on the equation by Janssen et al: ${ }^{17}$ skeletal muscle mass (kilogram) $=\left[\left(\right.\right.$ height $^{2}(\mathrm{~cm}) /$ bioelectrical resistance $(\mathrm{ohms}) \times 0.401)+(\operatorname{sex} \times 3.825)+$ (age $($ years $) \times-0.071)]+5.102$, where for sex, men $=1$ and women $=0$. Skeletal muscle mass was divided by body weight to obtain SMI. We used the definition of Janssen et $\mathrm{al}^{18}$ who set the SMI values of participants aged 18-39 years from the NHANES III database as the reference value and classified sarcopenia when the SMI values of a subject were below one standard deviation (SD) less than young adult values. Participants with BMI over $30 \mathrm{~kg} / \mathrm{m}^{2}$ and SMI below the one standard deviation cutoff were classified as having sarcopenic obesity.

Gait speed was recorded as a 20 -foot timed walk test in the mobile exam center following the NHANES standardized protocol. Gait speed was calculated as 6.15 meters (20 feet) divided by time (seconds).

\section{Covariates}

Demographic covariates were acquired from a self-report questionnaire that included age, sex, race, smoking history and comorbidities. Participants were considered smokers if they had smoked at least 20 cigarettes in their lives. Comorbidities were defined from self-report diagnoses, including diabetes, asthma and arthritis.

CRP was measured by latex-enhanced nephelometry. Fasting glucose was measured by a modified enzyme assay. Insulin was measured by radioimmunoassay. Total protein, total cholesterol, high-density lipoprotein (HDL) cholesterol and total triglyceride levels were quantified by a Hitachi 
Model 737 multichannel analyzer (Boehringer Mannheim Diagnostics, Indianapolis, IN). Low-density lipoprotein (LDL) cholesterol was computed by the Friedewald equation.

\section{Statistical Analyses}

We classified the study participants into 4 groups: group $\mathrm{N}$ (normal BMI population), group $\mathrm{S}$ (sarcopenia), group $\mathrm{O}$ (obesity) and group SO (sarcopenic obesity). Continuous measures, including age, BMI, SMI, gait speeds and laboratory data, are expressed as mean \pm SD. Comparison of continuous measures among participants in 4 groups was performed using one-way ANOVA. Categorical measures, including race, sex, smoking history and comorbidities, are expressed as percentages. Comparison of categorical measures among the 4 groups was performed using the chi-square test. The 4 groups were treated as independent variables and serum leptin level was used as the dependent variable. To assess the relationship between serum leptin level and sarcopenic obesity, we used multivariate regression analysis and developed 4 models to adjust for confounding variables. Model 1 was unadjusted. Model 2 included age, sex and race/ethnicity. Model 3 included Model 2 and BMI, serum C-reactive protein and fasting glucose. Model 4 included Model 3 and smoking history, arthritis, asthma and diabetes mellitus. All statistical analysis was done by using SPSS version 18 (SPSS Inc., Chicago, IL, USA).

\section{Results}

\section{Characteristics of the Study Population}

A total of 4063 participants were enrolled in the study: 178 (4.3\%) were classified as sarcopenia, 882 (21.7\%) as obesity and $75(1.8 \%)$ as sarcopenic obesity (Table 1$)$. The mean age of group $\mathrm{N}$ was $72.01 \pm 8.15$ years and $48.6 \%$ were male. For groups $\mathrm{S}, \mathrm{O}$ and $\mathrm{SO}$, the mean ages were $71.85 \pm 8.05$ years, $69.3 \pm 6.68$ years and $69.47 \pm 7.51$ years, respectively. Compared to group $\mathrm{N}$, the SMIs of group $\mathrm{S}$ and group SO were $19.83 \pm 2.99 \%$ and $14.21 \pm 2.6 \%$, respectively. Higher serum leptin level was found in group $\mathrm{O}$ and group $\mathrm{SO}$ than group $\mathrm{N}$ (p value $<0.001$ ).

\section{Association Between Serum Leptin Level and Components of Sarcopenic Obesity}

As shown in Table 2, we used multivariate regression models to investigate the association between serum leptin levels and components of sarcopenic obesity. A positive relationship between serum leptin levels and BMI was demonstrated in adjusted model ( $\beta$ coefficient: $1.02,95 \%$ confidence interval $(95 \% \mathrm{CI}): 0.79$ to $1.24, \mathrm{p}$ value $<0.001)$. Serum leptin level was inversely associated with SMI in the adjusted model ( $\beta$ coefficients: $-0.1,95 \% \mathrm{CI}$ : -0.17 to -0.02 , $\mathrm{p}$ value $=0.009$ ).

\section{Association Between Serum Leptin Level and 4 Groups (Normal BMI Population, Sarcopenia, Obesity and Sarcopenic Obesity)}

We explored the relationship between serum leptin level and 4 groups by performing multivariate regression models (Table 3). After adjustment for covariates, serum leptin level was positively associated with group $\mathrm{O}$ ( $\beta$ coefficients: $1.99,95 \% \mathrm{CI}: 0.16$ to 3.83 , p value $=0.032$ ) and group SO ( $\beta$ coefficients: $6.95,95 \% \mathrm{CI}: 2.59$ to 11.32 , $\mathrm{p}$ value $=0.002$ ).

\section{Discussion}

In this study, serum leptin level was significantly positively correlated with sarcopenic obesity risk. We also found a positive association between serum leptin levels and BMI. In contrast, serum leptin level was negatively correlated with SMI in multivariate regression models.

Our results corroborate the findings of previous studies. ${ }^{19-21}$ In the US study reported by Waters et al, baseline leptin level was higher in participants with sarcopenia, obesity and sarcopenic obesity. ${ }^{21}$ A crosssectional study from Japan revealed that serum leptin level was negatively associated with thigh muscle crosssectional area and positively correlated with visceral obesity, after adjusting for age, body weight and visceral fat area. ${ }^{19}$ A positive link between serum leptin levels and sarcopenic obesity has also been found in osteoarthritis patients. ${ }^{20}$ Higher levels of leptin and inflammatory cytokines (eg, IL-6 and TNF- $\alpha$ ) have been correlated with a greater risk of sarcopenia. ${ }^{8}$ However, previous studies of leptin impacts on sarcopenia and obesity have been conflicting. The study of Gómez et al revealed that serum leptin level was positively associated with fat-free mass. ${ }^{22}$ In a single-center observational study of hemodialysis patients, Lin et al noted an inverse association between serum leptin level and sarcopenia. ${ }^{23}$ Compared to healthy participants, frail hospitalized patients with a lower mid-arm muscle area had lower leptin. ${ }^{24}$ The cause of the discrepancies in 
Table I Characteristics of the Study Population

\begin{tabular}{|c|c|c|c|c|c|}
\hline Characteristic & $\begin{array}{l}\text { Normal Population } \\
(\mathrm{N}=\mathbf{2 9 2 8})\end{array}$ & Sarcopenia $(N=178)$ & Obesity ( $\mathbf{N = 8 8 2 )}$ & $\begin{array}{l}\text { Sarcopenic Obesity } \\
(\mathrm{N}=75)\end{array}$ & $P$ \\
\hline \multicolumn{6}{|l|}{ Socio-demographic variables } \\
\hline Age (years $\pm S D) *$ & $72.01(8.15)$ & $71.85(8.05)$ & $69.3(6.68)$ & $69.47(7.5 \mathrm{I})$ & $<0.001$ \\
\hline Male sex $(\%)^{\dagger}$ & $1423(48.6)$ & $70(39.3)$ & $534(60.5)$ & $4 \mid(54.7)$ & $<0.001$ \\
\hline Race-ethnicity $(\%)^{\dagger}$ & & & & & $<0.001$ \\
\hline Non-Hispanic White & $1832(62.6)$ & $100(56.2)$ & $466(52.8)$ & $37(49.3)$ & \\
\hline Smoker $(\%)$ I ever smoke $>20$ cigars in life & $516(17.6)$ & $37(20.8)$ & |3| (14.9) & $10(13.3)$ & 0.104 \\
\hline $\mathrm{BMI}\left(\mathrm{kg} / \mathrm{m}^{2} \pm \mathrm{SD}\right)^{*}$ & $25.02(3.07)$ & $25.18(2.67)$ & $33.92(3.83)$ & $34.5 I(4.7 I)$ & $<0.001$ \\
\hline Waist circumference $(\mathrm{cm} \pm \mathrm{SD})^{*}$ & $93.08(10.02)$ & $94.53(8.94)$ & II I.36 (9.48) & $113.66(10.62)$ & $<0.001$ \\
\hline Thigh skinfold (mm $\pm \mathrm{SD}$ )* & $246.69(1307.95)$ & $547.94(2082.28)$ & $1475.25(2630.89)$ & 1310.61 (2667.74) & $<0.001$ \\
\hline Skeletal muscle index $(\% \pm S D) *$ & $48.07(7.17)$ & $19.83(2.99)$ & $38.55(5.56)$ & $|4.2|(2.6)$ & $<0.001$ \\
\hline Gait speed $(\mathrm{m} / \mathrm{s} \pm \mathrm{SD})^{*}$ & $0.72(0.21)$ & $0.69(0.23)$ & $0.68(0.2)$ & $0.64(0.23)$ & $<0.001$ \\
\hline \multicolumn{6}{|l|}{ Laboratory variables* } \\
\hline Leptin (mg/dL $\pm S D)$ & $10.03(8)$ & $8.12(6.56)$ & $23.09(14.72)$ & $27.08(18.04)$ & $<0.001$ \\
\hline $\mathrm{CRP}(\mathrm{mg} / \mathrm{dL} \pm \mathrm{SD})$ & $0.53(0.99)$ & $0.58(1.02)$ & $0.65(0.89)$ & $0.67(1.1)$ & 0.009 \\
\hline Fasting Glucose $(\mathrm{mg} / \mathrm{dL} \pm \mathrm{SD})$ & $107.72(42.81)$ & $106.79(44.26)$ & I $18.96(49.33)$ & I $19.92(50.09)$ & $<0.001$ \\
\hline Insulin (uU/mL $\pm S D)$ & $59,597.74(222,362.02)$ & $71,144.12(242,354.44)$ & $63,105.75(228,496.1)$ & $77,304.91 \quad(253,227.57)$ & 0.909 \\
\hline Total protein (g/dL $\pm S D)$ & $7.3(0.48)$ & $7.3(0.52)$ & $7.35(0.47)$ & $7.33(0.56)$ & 0.061 \\
\hline LDL cholesterol $(\mathrm{mg} / \mathrm{dL} \pm \mathrm{SD})$ & $139.92(38.75)$ & $137.99(38.67)$ & $146.3(40.61)$ & $140.7(45.88)$ & 0.042 \\
\hline \multicolumn{6}{|l|}{ Comorbidities $^{\dagger}$} \\
\hline Asthma (\%) & $175(6)$ & $7(3.9)$ & $68(7.7)$ & $8(10.7)$ & $<0.001$ \\
\hline Arthritis (\%) & $1212(41.4)$ & $75(42.1)$ & $468(53.1)$ & $38(50.7)$ & $<0.001$ \\
\hline DM (\%) & $368(12.6)$ & $26(14.6)$ & $187(2 \mid .2)$ & $12(16)$ & $<0.001$ \\
\hline
\end{tabular}

Notes: *Continuous variables are presented as mean (standard deviation). ${ }^{\dagger}$ Categorical variables are presented as number (percentage).

Abbreviations: SD, standard deviation; BMI, body mass index; CRP, C-reactive protein; DM, diabetes mellitus; LDL, low-density lipoprotein.

Table 2 Regression Coefficients for Serum Leptin Levels and Components of Sarcopenic Obesity

\begin{tabular}{|l|l|l|l|l|l|l|l|l|}
\hline & \multicolumn{2}{|l|}{ Model I } & \multicolumn{2}{l|}{ Model 2 } & \multicolumn{2}{l|}{ Model 3 } & \multicolumn{2}{l|}{ Model 4 } \\
\cline { 2 - 9 } & $\boldsymbol{\beta} \mathbf{( 9 5 \% ~ C l )}$ & $\boldsymbol{P}$ & $\boldsymbol{\beta} \mathbf{( 9 5 \% ~ C l )}$ & $\boldsymbol{P}$ & $\boldsymbol{\beta} \mathbf{( 9 5 \% ~ C l )}$ & $\boldsymbol{P}$ & $\boldsymbol{\beta} \mathbf{( 9 5 \% ~ C l )}$ \\
\hline BMI & $1.35(1.23,1.46)$ & $<0.001$ & $1.25(1.15,1.35)$ & $<0.001$ & $1.01(0.79,1.23)$ & $<0.001$ & $1.02(0.79,1.24)$ & $<0.001$ \\
Skeletal muscle index & $-0.67(-0.74,-0.6)$ & $<0.001$ & $-0.52(-0.59,-0.45)$ & $<0.001$ & $-0.1(-0.17,-0.02)$ & 0.009 & $-0.1(-0.17,-0.02)$ & 0.009 \\
Gaitspeed & $-8.94(-12.71,-5.18)$ & $<0.001$ & $-7.19(-10.68,-3.69)$ & $<0.001$ & $-0.05(-2.76,2.65)$ & 0.966 & $-0.23(-2.95,2.48)$ & 0.866 \\
\hline
\end{tabular}

Notes: Adjusted covariates: Model I= unadjusted, Model 2= age, gender, race/ethnicity, Model 3= Model 2+ BMI, serum C-reactive protein, fasting glucose, waist circumference, thigh skinfold, serum insulin, total protein and low-density lipoprotein cholesterol, Model $4=$ Model $3+$ (smoking, arthritis, asthma and diabetes mellitus). Abbreviation: BMI, body mass index.

Table 3 Regression Coefficients for Serum Leptin Levels and 4 Groups

\begin{tabular}{|c|c|c|c|c|c|c|c|c|}
\hline & \multicolumn{2}{|l|}{ Model I } & \multicolumn{2}{|l|}{ Model 2} & \multicolumn{2}{|l|}{ Model 3} & \multicolumn{2}{|l|}{ Model 4} \\
\hline & $\beta(95 \% \mathrm{Cl})$ & $P$ & $\beta(95 \% \mathrm{Cl})$ & $P$ & $\beta(95 \% \mathrm{Cl})$ & $P$ & $\beta(95 \% \mathrm{Cl})$ & $P$ \\
\hline Normal & Reference(-,-) & & Reference(-,-) & & Reference(-,-) & & Reference(-,-) & \\
\hline Sarcopenia & $-0.89(-4.98,3.2)$ & 0.669 & $0.07(-3.43,3.57)$ & 0.969 & $-0.32(-3.4 I, 2.75)$ & 0.834 & $-0.3(-3.39,2.78)$ & 0.849 \\
\hline Obesity & $13.78(\mid 2.21,15.35)$ & $<0.001$ & $12.14(10.77,13.5 I)$ & $<0.001$ & $2.05(0.23,3.87)$ & 0.027 & $1.99(0.16,3.83)$ & 0.032 \\
\hline Sarcopenic obesity & $19.22(13.72,24.72)$ & $<0.001$ & $17.12(|2.4|, 21.83)$ & $<0.001$ & $6.97(2.61,11.33)$ & 0.002 & $6.95(2.59,|1| .32)$ & 0.002 \\
\hline
\end{tabular}

Notes: Adjusted covariates: Model I= unadjusted, Model 2= age, gender, race/ethnicity, Model 3= Model 2+ BMI, serum C-reactive protein and fasting glucose, waist circumference, thigh skinfold, serum insulin, total protein and low-density lipoprotein cholesterol, Model 4= Model 3+ (smoking, arthritis, asthma and diabetes mellitus).

these studies may be disparate effects of leptin on different populations such as obese individuals, hemodialysis subjects, elderly people, Europeans and Asians.
The circulating leptin level reflects the amount of adipose tissue, so there are higher levels of leptin in obese subjects. $^{25}$ Nevertheless, high leptin levels did not 
represent better efficacy of leptin, including affecting food consumption, lipid metabolism and glucose hemostasis. $^{25,26}$ The impairment of leptin function, as leptin resistance, was found to be associated with obesity. ${ }^{26,27}$ Previous studies shown that exogenous leptin administration to obese subjects was unable to affect weight loss. ${ }^{26-28}$ Obesity was correlated with reduced leptin receptors. ${ }^{29,30}$ This evidence indicated leptin resistance in obese individuals. ${ }^{26}$ Furthermore, increased leptin levels may induce the production of inflammatory cytokines, such as IL- 6 and TNF- $\alpha .^{31}$ Elevated inflammatory cytokines are known to be a pivotal factor of insulin resistance which is detrimental to muscle mass, quality and strength. ${ }^{32,33}$ Insulin resistance is a key pathway of sarcopenic obesity. ${ }^{12}$ High levels of leptin were proven to impact intramuscular fatty acid oxidation and lipid metabolism, thereby leading to skeletal muscle dysfunction. ${ }^{11,34}$ Impaired fatty acid oxidation and intramyocellular lipid accumulation may promote lipotoxicity. $^{12,35}$ Taken together, these studies and our findings suggest leptin may play a potential role in the mechanism of sarcopenic obesity.

Prior studies have proposed several definitions of sarcopenic obesity. ${ }^{36}$ Baumgartner defined sarcopenia as an appendicular skeletal muscle mass divided by height squared lower than 2 SD less than the sex-specific means and defined obesity as a percentage body fat higher than $27 \%$ in men and $38 \%$ in women. ${ }^{14}$ Davison et al defined sarcopenia as the lower two quintiles of full body skeletal muscle mass and obesity as the upper two quintiles of body fat. ${ }^{37}$ Recent studies have combined individual definitions of sarcopenia and obesity to define sarcopenic obesity. ${ }^{12}$ The European Working Group for the Study of Sarcopenia stated a definition for sarcopenia that included low muscle mass and function (handgrip strength or gait speed). ${ }^{38}$ The World Health Organization defines obesity as $\mathrm{BMI} \geq 30 \mathrm{~kg} / \mathrm{m} 2$ and visceral fat surrogate as waist circumference $\left(\mathrm{men} \geq 102 \mathrm{~cm}\right.$ and women $\geq 88 \mathrm{~cm}$ ). ${ }^{39}$ The American Association of Clinical Endocrinology suggests obesity as the percentage body fat $>25 \%$ for men and $>35 \%$ for women. ${ }^{40}$ A cross-sectional study by Batsis et al using eight definitions of sarcopenic obesity revealed a high degree of variation in prevalence rates in an older population. ${ }^{41}$ A Korean study also reported that different definitions of sarcopenic obesity affect prevalence estimates. $^{42}$ A consensus definition of sarcopenic obesity is needed for future observational studies or interventional trials.
Our study clearly has some limitations. First, the crosssectional design of the study does not allow us to assume a causal relationship between serum leptin level and sarcopenic obesity. Second, the observational nature of our study might be affected by uncontrolled covariates. We did not adjust for relevant proinflammatory cytokines and adipokines in sarcopenic obesity due to lack of data in NHNAES III database. Third, there is no universal standard definition of sarcopenic obesity. ${ }^{12}$ We used SMI $<1$ $\mathrm{SD}$ based on the Janssen definition of sarcopenia. We did not incorporate muscle strength or gait speed into the definition of sarcopenia which is commonly used.

\section{Conclusion}

In this study, we demonstrated that serum leptin level was positively associated with BMI and negatively associated with SMI in multivariate regression models. We also found a positive relationship between serum leptin levels and sarcopenic obesity in female subjects. Our findings provide epidemiologic evidence that leptin may be a risk factor for sarcopenic obesity. Prospective studies are warranted to address the causative relationship between serum leptin level and sarcopenic obesity.

\section{Abbreviation}

SMI, skeletal muscle index; BMI, body mass index; IL-6, interleukin-6; IL-12, interleukin-12; TNF- $\alpha$, tumor necrosis factor- $\alpha$; N, normal BMI population; S, sarcopenia; O, obesity; SO, sarcopenic obesity; NCHS, National Center for Health Statistics; NHANES III, the third National Health and Nutrition Examination Survey; HDL, highdensity lipoprotein; LDL, low-density lipoprotein; CRP, C-reactive protein.

\section{Author Contributions}

All authors made a significant contribution to the work reported, whether that is in the conception, study design, execution, acquisition of data, analysis and interpretation, or in all these areas; took part in drafting, revising or critically reviewing the article; gave final approval of the version to be published; have agreed on the journal to which the article has been submitted; and agree to be accountable for all aspects of the work.

\section{Role of the Funding Source}

There was no funding directly for this manuscript. 


\section{Disclosure}

There is no conflict of interest.

\section{References}

1. Kershaw EE, Flier JS. Adipose tissue as an endocrine organ. J Clin Endocrinol Metab. 2004;89(6):2548-2556. doi:10.1210/jc.2004-0395

2. Considine RV, Sinha MK, Heiman ML, et al. Serum immunoreactive-leptin concentrations in normal-weight and obese humans. $N$ Engl J Med. 1996;334(5):292-295. doi:10.1056/ nejm199602013340503

3. Morrison CD. Leptin signaling in brain: a link between nutrition and cognition? Biochim Biophys Acta. 2009;1792(5):401-408. doi:10.1016/j.bbadis.2008.12.004

4. Bjørbaek C, Kahn BB. Leptin signaling in the central nervous system and the periphery. Recent Prog Horm Res. 2004;59(1):305-331. doi:10.1210/rp.59.1.305

5. Kwon H, Pessin JE. Adipokines mediate inflammation and insulin resistance. Front Endocrinol. 2013;4:71. doi:10.3389/fendo.20 13.00071

6. Tilg H, Moschen AR. Adipocytokines: mediators linking adipose tissue, inflammation and immunity. Nat Rev Immunol. 2006;6 (10):772-783. doi:10.1038/nri1937

7. Sweeney G. Cardiovascular effects of leptin. Nat Rev Cardiol. 2010;7 (1):22-29. doi:10.1038/nrcardio.2009.224

8. Li CW, Yu K, Shyh-Chang N, et al. Circulating factors associated with sarcopenia during ageing and after intensive lifestyle intervention. J Cachexia Sarcopenia Muscle. 2019;10(3):586-600. doi: $10.1002 / \mathrm{jcsm} .12417$

9. Gregg EW, Cheng YJ, Cadwell BL, et al. Secular trends in cardiovascular disease risk factors according to body mass index in US adults. JAMA. 2005;293(15):1868-1874. doi:10.1001/jama.293.1 5.1868

10. Flegal KM, Kruszon-Moran D, Carroll MD, Fryar CD, Ogden CL. Trends in obesity among adults in the United States, 2005 to 2014. JAMA. 2016;315(21):2284-2291. doi:10.1001/jama.2016.6458

11. Kalinkovich A, Livshits G. Sarcopenic obesity or obese sarcopenia: a cross talk between age-associated adipose tissue and skeletal muscle inflammation as a main mechanism of the pathogenesis. Ageing Res Rev. 2017;35:200-221. doi:10.1016/j.arr.2016.09.008

12. Batsis JA, Villareal DT. Sarcopenic obesity in older adults: aetiology, epidemiology and treatment strategies. Nat Rev Endocrinol. 2018;14 (9):513-537. doi:10.1038/s41574-018-0062-9

13. Shafiee G, Keshtkar A, Soltani A, Ahadi Z, Larijani B, Heshmat R. Prevalence of sarcopenia in the world: a systematic review and metaanalysis of general population studies. J Diabetes Metab Disord. 2017;16(1):21. doi:10.1186/s40200-017-0302-x

14. Baumgartner RN. Body composition in healthy aging. Ann N Y Acad Sci. 2000;904(1):437-448. doi:10.1111/j.1749-6632.2000.tb06498.x

15. Choi KM. Sarcopenia and sarcopenic obesity. Korean J Intern Med. 2016;31(6):1054-1060. doi:10.3904/kjim.2016.193

16. Schrager MA, Metter EJ, Simonsick E, et al. Sarcopenic obesity and inflammation in the InCHIANTI study. J Appl Physiol. 2007;102 (3):919-925. doi:10.1152/japplphysiol.00627.2006

17. Janssen I, Heymsfield SB, Baumgartner RN, Ross R. Estimation of skeletal muscle mass by bioelectrical impedance analysis. $J$ Appl Physiol. 2000;89(2):465-471. doi:10.1152/jappl.2000.89.2.465

18. Janssen I, Heymsfield SB, Ross R. Low relative skeletal muscle mass (sarcopenia) in older persons is associated with functional impairment and physical disability. J Am Geriatr Soc. 2002;50(5):889-896. doi:10.1046/j.1532-5415.2002.50216.x

19. Kohara K, Ochi M, Tabara Y, Nagai T, Igase M, Miki T. Leptin in sarcopenic visceral obesity: possible link between adipocytes and myocytes. PLoS One. 2011;6(9):e24633-e24633. doi:10.1371/journal.pone. 0024633
20. Manoy P, Anomasiri W, Yuktanandana P, et al. Elevated serum leptin levels are associated with low vitamin $\mathrm{D}$, sarcopenic obesity, poor muscle strength, and physical performance in knee osteoarthritis. Biomarkers. 2017;22(8):723-730. doi:10.1080/ 1354750x.2017.1315615

21. Waters DL, Qualls CR, Dorin RI, Veldhuis JD, Baumgartner RN. Altered growth hormone, cortisol, and leptin secretion in healthy elderly persons with sarcopenia and mixed body composition phenotypes. J Gerontol a Biol Sci Med Sci. 2008;63(5):536-541. doi:10.1093/gerona/63.5.536

22. Gómez JM, Maravall FJ, Gómez N, Navarro MA, Casamitjana R, Soler J. Interactions between serum leptin, the insulin-like growth factor-I system, and sex, age, anthropometric and body composition variables in a healthy population randomly selected. Clin Endocrinol. 2003;58(2):213-219. doi:10.1046/j.1365-2265.2003.01698.x

23. Lin YL, Wang CH, Lai YH, Kuo CH, Syu RJ, Hsu BG. Negative correlation between leptin serum levels and sarcopenia in hemodialysis patients. Int J Clin Exp Pathol. 2018;11(3):1715-1723.

24. Hubbard RE, O'Mahony MS, Calver BL, Woodhouse KW. Nutrition, inflammation, and leptin levels in aging and frailty. $J$ Am Geriatr Soc. 2008;56(2):279-284. doi:10.1111/j.1532-5415.2007.01548.x

25. Park HK, Ahima RS. Physiology of leptin: energy homeostasis, neuroendocrine function and metabolism. Metabolism. 2015;64 (1):24-34. doi:10.1016/j.metabol.2014.08.004

26. Farr OM, Gavrieli A, Mantzoros CS. Leptin applications in 2015: what have we learned about leptin and obesity? Curr Opin Endocrinol Diabetes Obes. 2015;22(5):353-359. doi:10.1097/ med.0000000000000184

27. Sáinz N, Barrenetxe J, Moreno-Aliaga MJ, Martínez JA. Leptin resistance and diet-induced obesity: central and peripheral actions of leptin. Metabolism. 2015;64(1):35-46. doi:10.1016/j. metabol.2014.10.015

28. Shetty GK, Matarese G, Magkos F, et al. Leptin administration to overweight and obese subjects for 6 months increases free leptin concentrations but does not alter circulating hormones of the thyroid and IGF axes during weight loss induced by a mild hypocaloric diet. Eur J Endocrinol. 2011;165(2):249-254. doi:10.1530/eje-11-0252

29. Fuentes T, Ara I, Guadalupe-Grau A, et al. Leptin receptor $170 \mathrm{kDa}$ (OB-R170) protein expression is reduced in obese human skeletal muscle: a potential mechanism of leptin resistance. Exp Physiol. 2010;95(1):160-171. doi:10.1113/expphysiol.2009.049270

30. Van dielen FMH, van 'T Veer C, Buurman WA, Greve JWM. Leptin and soluble leptin receptor levels in obese and weight-losing individuals. $J$ Clin Endocrinol Metab. 2002;87(4):1708-1716. doi:10.1210/jcem.87.4.8381

31. López-Jaramillo P, Gómez-Arbeláez D, López-López J, et al. The role of leptin/adiponectin ratio in metabolic syndrome and diabetes. Horm Mol Biol Clin Investig. 2014;18(1):37-45. doi:10.1515/hmbci2013-0053

32. Rehman K, Akash MSH. Mechanisms of inflammatory responses and development of insulin resistance: how are they interlinked? J Biomed Sci. 2016;23(1):87. doi:10.1186/s12929-016-0303-y

33. Yang J. Enhanced skeletal muscle for effective glucose homeostasis. Prog Mol Biol Transl Sci. 2014;121:133-163. doi:10.1016/b9780-12-800101-1.00005-3

34. Ceddia RB. Direct metabolic regulation in skeletal muscle and fat tissue by leptin: implications for glucose and fatty acids homeostasis. Int J Obes. 2005;29(10):1175-1183. doi:10.1038/sj.ijo.0803025

35. Lelliott C, Vidal-Puig AJ. Lipotoxicity, an imbalance between lipogenesis de novo and fatty acid oxidation. Int J Obes. 2004;28(4):S22S28. doi:10.1038/sj.ijo.0802854

36. Stenholm S, Harris TB, Rantanen T, Visser M, Kritchevsky SB, Ferrucci L. Sarcopenic obesity: definition, cause and consequences. Curr Opin Clin Nutr Metab Care. 2008;11(6):693-700. doi:10.1097/ MCO.0b013e328312c37d 
37. Davison KK, Ford ES, Cogswell ME, Dietz WH. Percentage of body fat and body mass index are associated with mobility limitations in people aged 70 and older from NHANES III. $J$ Am Geriatr Soc. 2002;50(11):1802-1809. doi:10.1046/j.1532-5415.2002.50508.x

38. Cruz-Jentoft AJ, Baeyens JP, Bauer JM, et al. Sarcopenia: European consensus on definition and diagnosis: report of the European Working Group on Sarcopenia in Older People. Age Ageing. 2010;39(4):412-423. doi:10.1093/ageing/afq034

39. World Health Organization. Obesity: preventing and managing the global epidemic. Report of a WHO consultation. World Health Organ Tech Rep Ser. 2000;894:i-xii, 1-253.

40. Garvey WT, Mechanick JI, Brett EM, et al. American Association of Clinical Endocrinologists and American College of Endocrinology comprehensive clinical practice guidelines for medical care of patients with obesity. Endocr Pract. 2016;22(Suppl 3):1-203. doi:10.4158/ep161365.Gl
41. Batsis JA, Barre LK, Mackenzie TA, Pratt SI, Lopez-Jimenez F, Bartels SJ. Variation in the prevalence of sarcopenia and sarcopenic obesity in older adults associated with different research definitions: dual-energy X-ray absorptiometry data from the National Health and Nutrition Examination Survey 1999-2004. J Am Geriatr Soc. 2013;61(6):974-980. doi:10.1111/jgs. 12260

42. Kim YS, Lee Y, Chung YS, et al. Prevalence of sarcopenia and sarcopenic obesity in the Korean population based on the Fourth Korean National Health and Nutritional Examination Surveys. $J$ Gerontol a Biol Sci Med Sci. 2012;67(10):1107-1113. doi:10.1093/gerona/gls071
Journal of Inflammation Research

\section{Publish your work in this journal}

The Journal of Inflammation Research is an international, peerreviewed open-access journal that welcomes laboratory and clinical findings on the molecular basis, cell biology and pharmacology of inflammation including original research, reviews, symposium reports, hypothesis formation and commentaries on: acute/chronic inflammation; mediators of inflammation; cellular processes; molecular
Dovepress

mechanisms; pharmacology and novel anti-inflammatory drugs; clinical conditions involving inflammation. The manuscript management system is completely online and includes a very quick and fair peerreview system. Visit http://www.dovepress.com/testimonials.php to read real quotes from published authors.

Submit your manuscript here: https://www.dovepress.com/journal-of-inflammation-research-journal 